\title{
PRE-CULTIVATION OF ADIPOSE TISSUE-DERIVED MICROVASCULAR FRAGMENTS IN POROUS SCAFFOLDS DOES NOT IMPROVE THEIR IN VIVO VASCULARISATION POTENTIAL
}

\author{
M.W. Laschke ${ }^{1,2, *}$, S. Kleer ${ }^{1,2}$, C. Scheuer ${ }^{1,2}$, D. Eglin ${ }^{3}$, M. Alini ${ }^{3}$ and M.D. Menger ${ }^{1,2}$ \\ ${ }^{1}$ Institute for Clinical \& Experimental Surgery, University of Saarland, 66421 Homburg/Saar, Germany \\ ${ }^{2}$ Collaborative Research Partner Large Bone Defect Healing Program of AO Foundation \\ ${ }^{3} \mathrm{AO}$ Research Institute Davos, Clavadelerstrasse, 7270 Davos Platz, Switzerland
}

\begin{abstract}
Adipose tissue-derived microvascular fragments represent promising vascularisation units for implanted tissue constructs. However, their reassembly into functional microvascular networks takes several days, during which the cells inside the implants are exposed to hypoxia. In the present study, we analysed whether this critical phase may be overcome by pre-cultivation of fragment-seeded scaffolds prior to their implantation. Green fluorescent protein (GFP)-positive microvascular fragments were isolated from epididymal fat pads of male C57BL/6-TgN (ACTB-EGFP) 1Osb/J mice. Nano-size hydroxyapatite particles/poly (ester-urethane) scaffolds were seeded with these fragments and cultivated for 28 days. Subsequently, these scaffolds or control scaffolds, which were freshly seeded with GFP-positive microvascular fragments, were implanted into the dorsal skinfold chamber of C57BL/6 wild-type mice to study their vascularisation and incorporation by means of intravital fluorescence microscopy, histology and immunohistochemistry over 2 weeks. Pre-cultivation of microvascular fragments resulted in the loss of their native vessel morphology. Accordingly, pre-cultivated scaffolds contained a network of individual CD31/GFP-positive endothelial cells with filigrane cell protuberances. After implantation into the dorsal skinfold chamber, these scaffolds exhibited an impaired vascularisation, as indicated by a significantly reduced functional microvessel density and lower fraction of GFPpositive microvessels in their centre when compared to freshly seeded control implants. This was associated with a deteriorated incorporation into the surrounding host tissue. These findings indicate that freshly isolated, noncultivated microvascular fragments should be preferred as vascularisation units. This would also facilitate their use in clinical practice during intra-operative one-step procedures.
\end{abstract}

Keywords: Tissue engineering, microvascular fragments, pre-cultivation, adipose tissue, vascularisation, inosculation, scaffold, polyurethane

*Address for correspondence:

Matthias W. Laschke, MD, PhD

Institute for Clinical \& Experimental Surgery

University of Saarland, 66421 Homburg/Saar, Germany

Telephone number: +496841 1626554

Fax number: +496841 1626553

E-mail: matthias.laschke@uks.eu

\section{Introduction}

The success of tissue engineering approaches depends on the establishment of an adequate vascularisation, which provides the basis for the onset of regenerative processes and guarantees the survival of cell-seeded implants (Laschke et al., 2006; Auger et al., 2013). Accordingly, various vascularisation strategies have been tested during the last years, ranging from the stimulation of angiogenesis at the defect site by sophisticated growth factor delivery systems to the generation of preformed microvascular networks inside tissue constructs prior to their implantation (Phelps and García, 2010; Novosel et al., 2011). By now, particularly the latter approach is considered to be a promising option, because the rapid interconnection of preformed microvessels with the surrounding microvasculature may markedly shorten the critical time period, during which an implant is exposed to hypoxia (Laschke et al., 2009; Laschke and Menger, 2012).

Adipose tissue-derived stromal cells represent an attractive source for the generation of prevascularised tissue constructs due to their high vessel-forming capacity (Scherberich et al., 2010). This heterogeneous population of isolated multipotent stem cells, endothelial cells, pericytes and haematopoietic cells has been shown to assemble into microvascular networks when seeded onto different scaffold types (Scherberich et al., 2007; Müller et al., 2010; Güven et al., 2011; Laschke et al., 2013; Klar et al., 2014). Moreover, the cells promote angiogenesis and vessel maturation by secreting various pro-angiogenic and regulatory proteins (Matsuda et al., 2013; Rohringer et al., 2014).

Alternatively to adipose tissue-derived single cell suspensions, intact microvascular fragments may be used as effective prevascularisation units for tissue constructs (Laschke et al., 2012; Laschke et al., 2014a). These mixed arteriolar, capillary and venular vessel segments can also be easily isolated in large amounts from fat tissue samples by enzymatic digestion within a short period of time (Hoying et al., 1996; Shepherd et al., 2004; Nunes et al., 2010a). Importantly, they do not only exhibit a fully functional vessel morphology directly after isolation, but also contain mesenchymal stem cells and endothelial progenitor cells, which may further improve their regenerative potential (Laschke et al., 2012; McDaniel et al., 2014). Accordingly, microvascular fragments have already been successfully used to improve the perfusion of different tissue constructs, including muscle, cardiac and islet implants (Shepherd et al., 2007; Hiscox et al., 2008; Pilia et al., 2014).

After their incorporation into porous scaffolds, microvascular fragments exhibit a high angiogenic 
activity and reassemble in vivo to functional microvascular networks, which are finally blood-perfused by the process of external inosculation (Laschke et al., 2012). However, although this approach circumvents de novo vessel formation inside the scaffolds, it still does not result in their sufficient blood perfusion during the first 3-6 days after implantation. This is because the formation of a new microvasculature, based on microvascular fragments, also requires a time-consuming sequence of distinct vascular phenotypes, including sprouting angiogenesis, neovascular formation and network maturation (Nunes et al., 2010b).

To overcome this problem, we analysed in the present study whether it is possible to generate complete microvascular networks by pre-cultivation of microvascular fragments inside porous scaffolds under pro-angiogenic culture conditions. We hypothesised that these networks are already in a mature stage at the time point of implantation and, thus, simply have to develop interconnections to the surrounding vessels to get fully perfused within a short period of time. To test this hypothesis, we implanted pre-cultivated scaffolds and control scaffolds, which were freshly seeded with microvascular fragments, into mouse dorsal skinfold chambers to analyse their vascularisation and incorporation by means of repetitive intravital fluorescence microscopy, histology and immunohistochemistry.

\section{Materials and Methods}

\section{Animals}

For the experiments we used male transgenic C57BL/6TgN (ACTB-EGFP)1Osb/J mice (The Jackson Laboratory, Maine, USA) with an average age of 8 months and a body weight of 30-36 $\mathrm{g}$ as fat donors and corresponding male C57BL/6 wild-type mice (Charles River, Sulzfeld, Germany) with an average age of 3-4 months and a body weight of 22-26 $\mathrm{g}$ for the preparation of dorsal skinfold chambers. The animals were housed one per cage and had free access to tap water and standard pellet food (Altromin, Lage, Germany). All experiments were approved by the local governmental animal care committee and were conducted in accordance with the German legislation on protection of animals and the NIH Guidelines for the Care and Use of Laboratory Animals (NIH Publication \#85-23 Rev. 1985).

\section{Isolation of microvascular fragments, seeding of scaffolds and pre-cultivation}

Microvascular fragments were isolated from epididymal fat pads of male C57BL/6-TgN (ACTB-EGFP)1Osb/J donor mice (Okabe et al., 1997), as described previously in detail (Laschke et al., 2012). This approach allowed an easy detection of the microvascular fragments by their GFP-positive signal throughout the experiments.

Directly after the isolation procedure, the microvascular fragments were seeded on porous nano-size hydroxyapatite particles/poly(ester-urethane) composite scaffolds with a size of $\sim 3 \times 3 \times 1 \mathrm{~mm}$, which were fabricated by a salt leaching-phase inverse process and exhibited interconnected macropores of $\sim 220 \mu \mathrm{m}$ (Laschke et al., 2010a). For this purpose, the scaffolds were fixed in the lumen of a modified $1 \mathrm{~mL}$ syringe (BD Plastipak; BD Biosciences, Heidelberg, Germany). The tip of the syringe was filled with $20 \mu \mathrm{L}$ PBS containing the microvascular fragments. Negative and positive pressure was alternately induced three to five times in the syringe so that the microvascular fragments could pass the scaffolds from both sides and were finally trapped in the scaffold pores (Laschke et al., 2012). Finally, the scaffolds were carefully taken out of the syringe and transferred into endothelial cell growth medium MV (PromoCell, Heidelberg, Germany) supplemented with $25 \mathrm{ng} / \mathrm{mL}$ vascular endothelial growth factor (VEGF; Promokine, Heidelberg, Germany) and $25 \mathrm{ng} / \mathrm{mL}$ fibroblast growth factor (FGF)- $\beta$ (Promokine). They were cultivated at $37^{\circ} \mathrm{C}$ in a humidified atmosphere with $5 \% \mathrm{CO}_{2}$ for $3,7,14$ or 28 days with medium change every 2 days. Subsequently, the scaffolds were embedded for histological analyses (samples from day 3, 7, 14 or 28 ) or implanted into dorsal skinfold chambers for further in vivo experiments (samples from day 28). As controls, we used freshly seeded scaffolds, which were not precultivated prior to their implantation.

\section{Dorsal skinfold chamber model}

Freshly seeded scaffolds and scaffolds, which were precultivated for 28 days, were implanted into the dorsal skinfold chamber of C57BL/6 wild-type mice to analyse their vascularisation by means of repetitive intravital fluorescence microscopy (Fig. 1a). For the preparation of the chamber, the animals were anaesthetised by intraperitoneal (i.p.) injection of ketamine $(75 \mathrm{mg} / \mathrm{kg}$ body weight; Ursotamin, Serumwerk Bernburg AG, Bernburg, Germany) and xylazine (25 mg/kg body weight; Rompun, Bayer, Leverkusen, Germany). A detailed description of the stepwise preparation of the dorsal skinfold chamber has been given previously by Laschke et al. (2011a). After the surgical procedure the animals were allowed to recover for $48 \mathrm{~h}$ in order to exclude deterioration of the microcirculation due to anaesthesia and surgical trauma. Subsequently, the cover glass of the dorsal skinfold chamber was temporarily removed and one scaffold was placed onto the striated muscle tissue within the centre of each chamber, taking care to avoid contamination, mechanical irritation or damage of the tissue (Fig. 1b).

\section{Intravital fluorescence microscopy and microcirculatory analysis}

For intravital fluorescence microscopy, $0.05 \mathrm{~mL} 5 \%$ fluorescein isothiocyanate (FITC)-labelled dextran 150,000 (Sigma-Aldrich, Taufkirchen, Germany) was injected intravenously (i.v.) via the retrobulbary space of the anaesthetised mice. This provided adequate contrast enhancement by staining of blood plasma. The animals were then fixed on a Plexiglas stage and the observation window of the chamber was horizontally positioned under the microscope. The microscopy was performed using a Zeiss Axiotech microscope (Zeiss, Oberkochen, Germany) with a $100 \mathrm{~W}$ mercury lamp, which was attached to an epi-illumination filter block for blue, green and ultraviolet light (Laschke et al., 2013). The microscopic images were recorded by a charge-coupled device video camera 

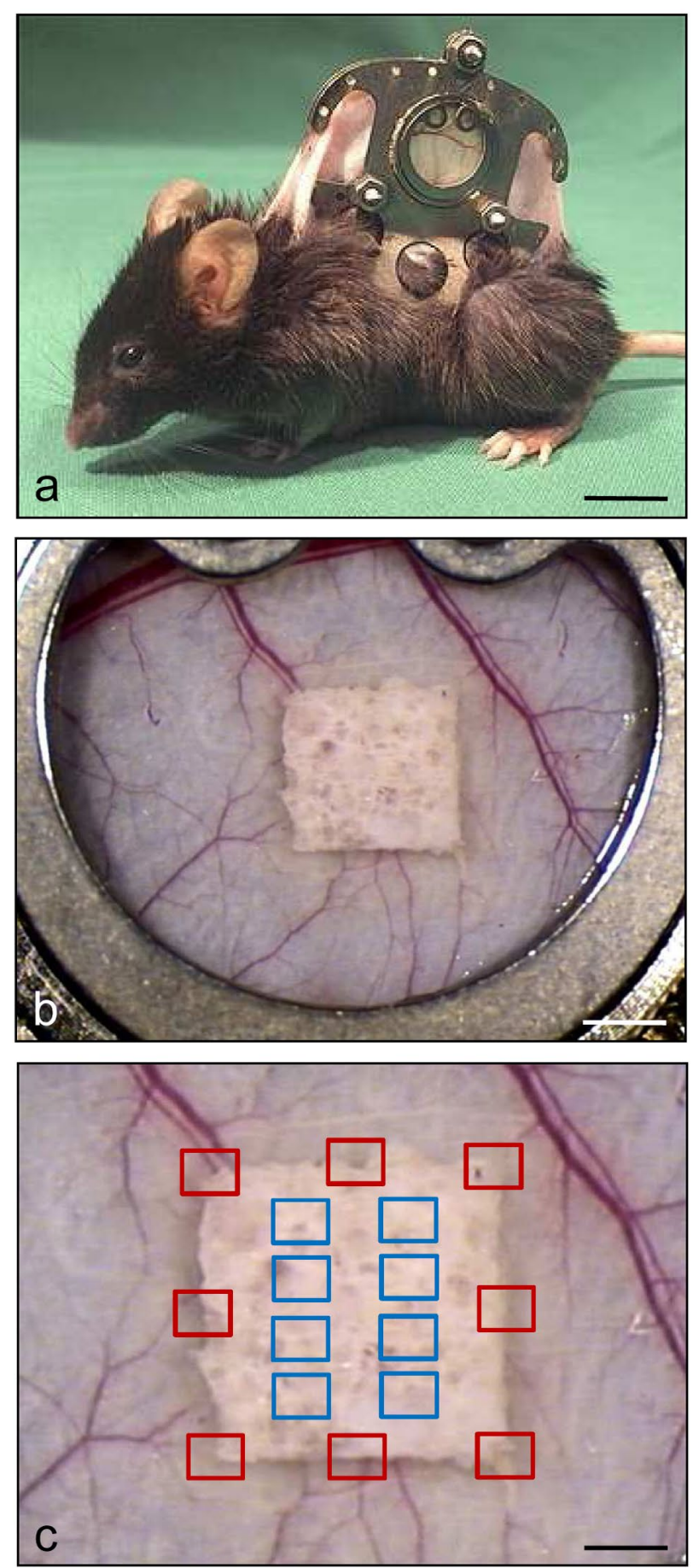

Fig. 1. a: C57BL/6 mouse equipped with a dorsal skinfold chamber (chamber weight $\sim 2 \mathrm{~g}$ ). b: Overview of the observation window of the chamber directly after implantation of a polyurethane scaffold $(\sim 3 \times 3 \times 1 \mathrm{~mm})$. c: Intravital fluorescent microscopic analyses included the assessment of vascularisation in 8 different ROIs within the border zone (red) and 8 different ROIs within the centre (blue) of the scaffolds. Scale bars: $\mathbf{a}=1.1 \mathrm{~cm}$; $\mathbf{b}=1.4 \mathrm{~mm} ; \mathbf{c}=850 \mu \mathrm{m}$.

(FK6990; Pieper, Schwerte, Germany) and transferred to a DVD system for off-line analysis. By means of $5 \times, 10 \times$ and $20 \times$ long-distance objectives (Zeiss) magnifications of $\times 115, \times 230$ and $\times 460$ were achieved on a 14 inch video screen (Trinitron; Sony, Tokyo, Japan).
The microscopic images were analysed by means of the software package CapImage (Zeintl, Heidelberg, Germany). Vascularisation of the scaffolds was evaluated at a magnification of $\times 460$ in 8 different microvascular regions of interest (ROIs) within the border zone and 8 different microvascular ROIs within the centre of the implants (Fig. 1c). Perfused ROIs (given in \% of all analysed ROIs) were defined as areas, which exhibited either newly developed red blood cell (RBC)-perfused microvessels or reperfused microvascular fragments. In addition, the functional microvessel densities, i.e. the lengths of all RBC-perfused blood vessels per ROI, were measured and are given in $\mathrm{cm} / \mathrm{cm}^{2}$ (Laschke et al., 2012).

\section{Experimental protocol}

Microvascular fragments were isolated from epididymal fat pads of 12 male C57BL/6-TgN(ACTB-EGFP)1Osb/J mice and seeded on 20 porous scaffolds. Directly after the seeding procedure $(n=4)$ or after 28 days of pre-cultivation $(n=4)$, serial sections of scaffolds were prepared for histological and immunohistochemical analyses to assess vessel distribution and morphology on 5 different sections per sample. The other scaffolds, which were pre-cultivated for $3(n=3), 7(n=3), 14(\mathrm{n}=3)$ and 28 days $(n=3)$ were additionally stained with trypan blue for the assessment of cell viability. For this purpose, the scaffolds were incubated in $0.2 \%$ trypan blue (Sigma-Aldrich) for $10 \mathrm{~min}$ at $37^{\circ} \mathrm{C}$. After washing in PBS for $10 \mathrm{~min}$, the samples were fixed in $4 \%$ formalin for further histological processing.

To analyse the vascularisation of the scaffolds in vivo, microvascular fragments from 12 male C57BL/6TgN(ACTB-EGFP) 1 Osb/J mice were seeded on 16 scaffolds, which were either pre-cultivated for 28 days $(n=8)$ or directly implanted $(n=8)$ into the dorsal skinfold chamber of 16 C57BL/6 wild-type mice. Intravital fluorescence microscopy of the implants' vascularisation was performed directly after implantation as well as at days $3,6,10$ and 14. At the end of the in vivo experiments, the animals were sacrificed with an overdose of the anaesthetic and the dorsal skinfold preparations were excised for further histological and immunohistochemical analyses.

\section{Histology and immunohistochemistry}

For light microscopy, formalin-fixed specimens of freshly seeded or pre-cultivated scaffolds as well as dorsal skinfold preparations with the scaffold implants were embedded in paraffin. $3 \mu \mathrm{m}$ sections were cut and stained with haematoxylin and eosin (HE) according to standard procedures. Sections of trypan blue-incubated scaffolds were counterstained with $0.1 \%$ nuclear fast red (Sigma-Aldrich) and examined under a BX60 microscope (Olympus, Hamburg, Germany) for the quantitative analysis of dead cells (marked in blue, given in \%) within the samples.

For immunohistochemical detection of endothelial cells and stabilising perivascular cells of microvascular fragments, sections were stained with a monoclonal rat-anti-mouse antibody against CD31 (1:30; dianova $\mathrm{GmbH}$, Hamburg, Germany) and with a rabbit-anti-mouse antibody against $\alpha$-smooth muscle actin (SMA) (1:100; Abcam, Cambridge, UK). A goat-anti-rat Cy3 antibody 

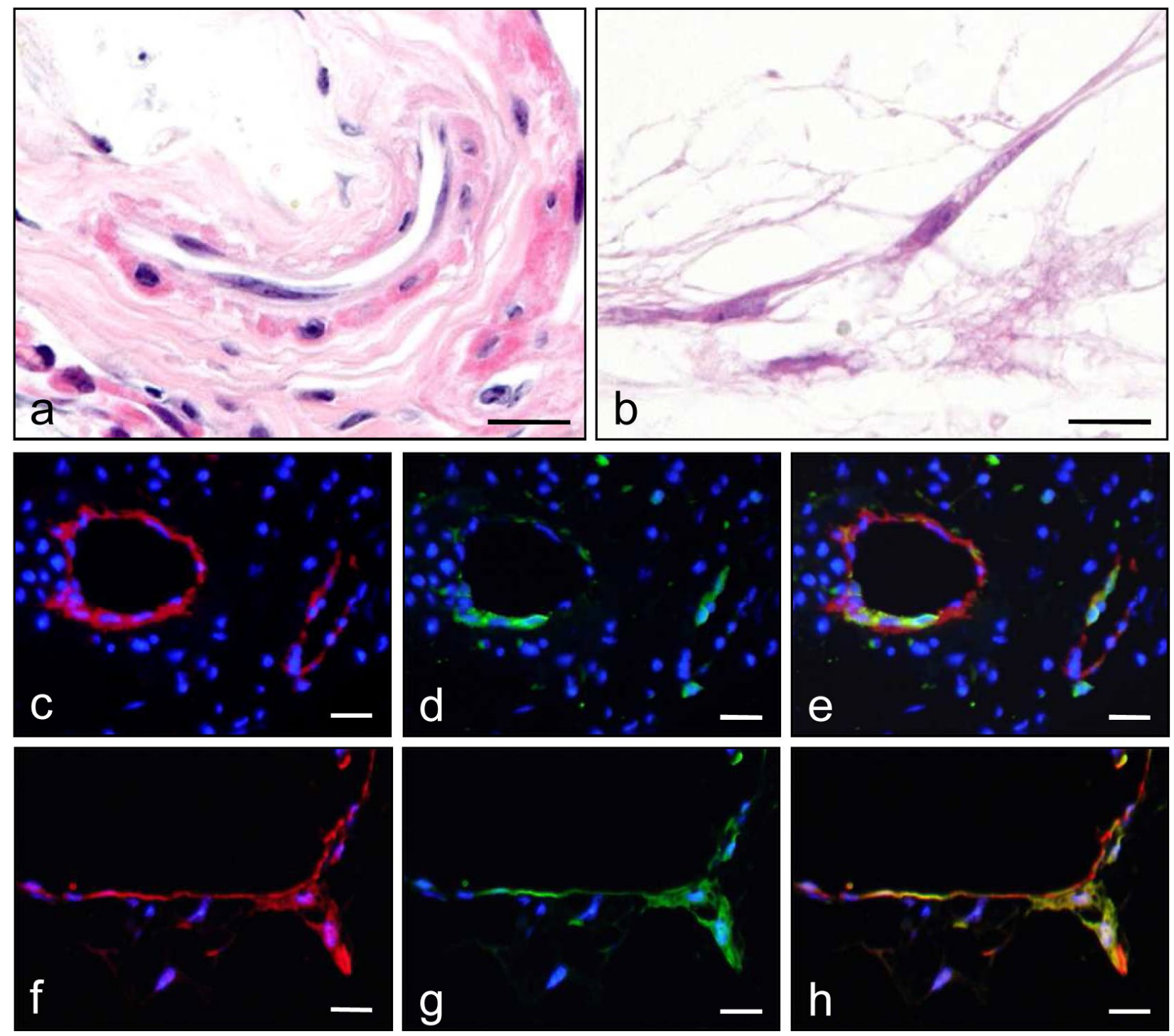

Fig. 2. a, b: HE-stained sections of a microvascular fragment with typical vessel morphology within a freshly seeded control scaffold (a) and thin, elongated cells, which grow along the scaffold strands of a pre-cultivated scaffold (b). c-h: Immunohistochemical characterisation of microvascular fragments within a control scaffold (c-e) and elongated cells in a pre-cultivated scaffold (f-h). Histological sections were stained with Hoechst 33342 to identify cell nuclei (c-h, blue), an antibody against CD31 for the detection of endothelial cells (c, f, red) and an antibody against GFP (d, $\mathbf{g}$, green). e and $\mathbf{h}$ display merges of (c, $\mathbf{d})$ and (f, $\mathbf{g})$. Scale bars: $\mathbf{a}, \mathbf{b}=20 \mu \mathrm{m} ; \mathbf{c}-\mathbf{h}=30 \mu \mathrm{m}$.

(1:50; dianova $\mathrm{GmbH})$ and a goat-anti-rabbit Alexa488 antibody (1:100; Invitrogen, Darmstadt, Germany) served as secondary antibodies. On each section, cell nuclei were stained with Hoechst 33342 (1:500; Sigma-Aldrich) to merge the images exactly.

For immunohistochemical detection of GFP-positive and -negative microvessels, sections were stained with a monoclonal rat-anti-mouse antibody against CD31 (1:30; dianova $\mathrm{GmbH})$ to detect endothelial cells and with a goat-anti-GFP antibody (1:200; Biomol, Hamburg, Germany) to enhance GFP-fluorescence. As secondary antibodies a goat-anti-rat $\mathrm{Cy} 3$ antibody (1:50; dianova $\mathrm{GmbH})$ and a biotin-labeled donkey-anti-goat antibody (1:15; Jackson ImmunoResearch, Baltimore, MD, USA), which was detected by fluorescein labelled-streptavidin (1:50; Vector Labs, Burlingame, CA, USA), were used. For this purpose, the sections were placed in Coplin jars with $0.05 \%$ citraconic anhydride solution $(\mathrm{pH} 7.4)$ for $1 \mathrm{~h}$ at $98^{\circ} \mathrm{C}$ and then incubated overnight at $4{ }^{\circ} \mathrm{C}$ with the primary antibody, followed by the appropriate secondary antibody at $37^{\circ} \mathrm{C}$ for $2 \mathrm{~h}$. On each section, cell nuclei were stained with Hoechst 33342 (1:500; Sigma-Aldrich) to merge the images exactly. For the quantitative analysis of the density (given in $\mathrm{mm}^{-2}$ ) and the fraction of GFP-positive and -negative microvessels (given in \%) in the border and centre of the implants, the sections were examined using the BX60 microscope (Olympus).

\section{Statistics}

After testing the data for normal distribution and equal variance, differences between two groups were analysed by the unpaired Student's $t$-test. To test for time effects in the individual groups, ANOVA for repeated measures was applied. This was followed by the Student-Newman-Keuls 

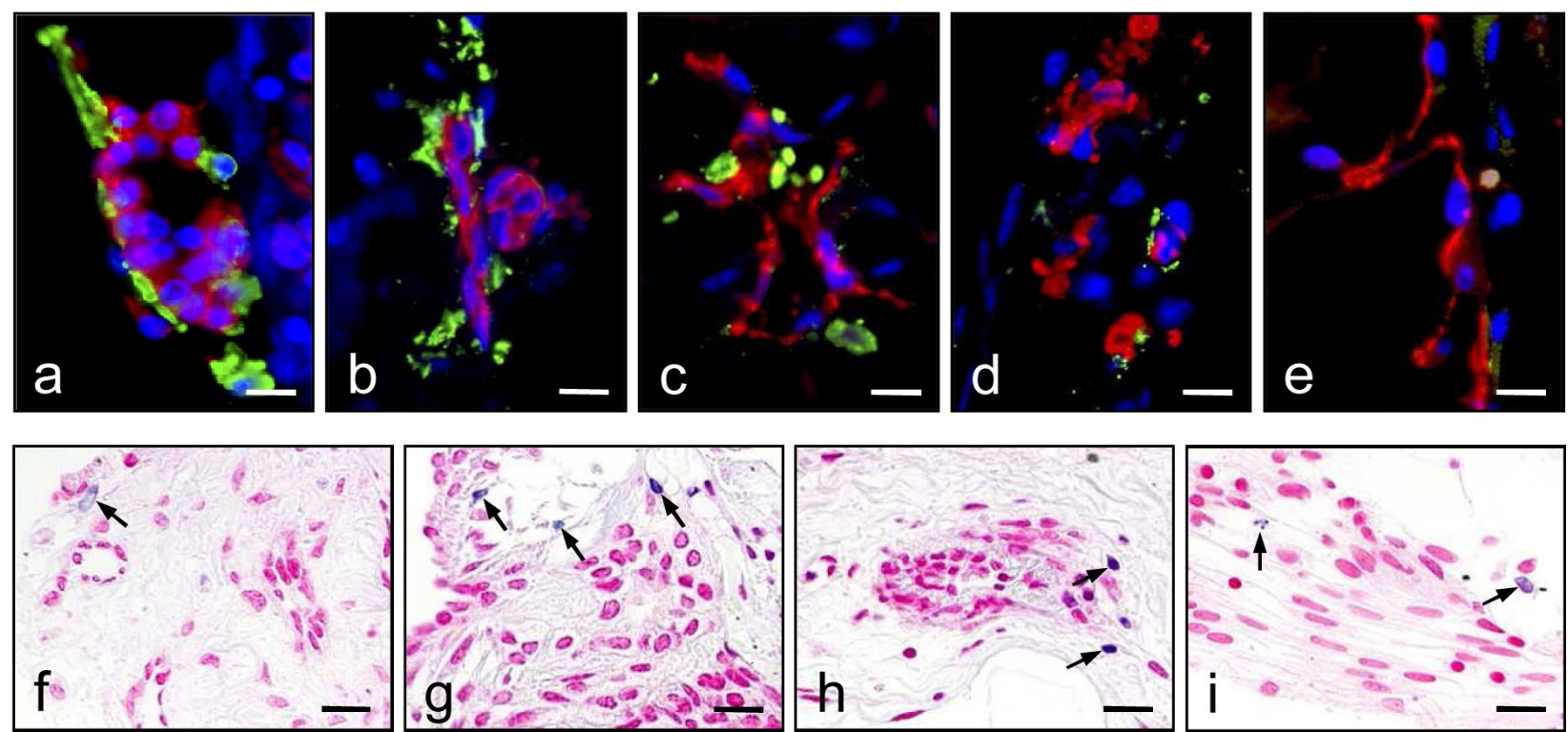

Fig. 3. a-e: Immunohistochemical characterisation of microvascular fragments within a freshly seeded control scaffold (a) and scaffolds, which were pre-cultivated for 3 (b), 7 (c), 14 (d) and 28 days (e). Note the continuous loss of the stabilising $\alpha$-SMA-positive perivascular cell coverage and the progressive disorganisation of the endothelial cells. Histological sections were stained with Hoechst 33342 to identify cell nuclei (a-e, blue), an antibody against CD31 for the detection of endothelial cells (a-e, red) and an antibody against $\alpha$-SMA (a-e, green). Scale bars: $15 \mu \mathrm{m}$. f-i: Nuclear fast red-stained sections of trypan blue-incubated scaffolds, which were pre-cultivated for 3 (f), 7 (g), 14 (h) or 28 days (i). Note that all samples only contain a very low number of trypan blue-positive dead cells (f-i, arrows). Scale bars: $\mathbf{f}-\mathbf{i}=20 \mu \mathrm{m}$.

test including the correction of the alpha error according to Bonferroni probabilities to compensate for multiple comparisons (SigmaStat; Jandel Corporation; San Rafael, CA, USA). All values are expressed as means \pm SEM. Statistical significance was accepted for a value of $p<0.05$.

\section{Results}

\section{Histomorphology of freshly seeded and pre-} cultivated scaffolds in vitro

In a first set of experiments, we characterised in vitro the morphology of freshly seeded and pre-cultivated scaffolds by means of histology and immunohistochemistry. These analyses revealed that the microvascular fragments were randomly distributed on the surface and inside central pores of freshly seeded control scaffolds. Because these fragments originated from mature microvascular networks of fat tissue samples, they comprised a heterogeneous mixture of arteriolar, venular and capillary segments, which were partly surrounded by residual connective tissue components and exhibited a typical vessel morphology with a lumen surrounded by an endothelial layer and perivascular cells (Fig. 2a). As expected, additional immunohistochemical analyses of the microvascular fragments showed that they stained double-positive for the endothelial cell marker CD31 and for GFP (Figs. 2c-e).

In contrast, scaffolds, which were pre-cultivated for 28 days, presented with a completely different histomorphology. They contained a network of thin, elongated cells, which grew along the scaffold strands (Fig. 2b). Of interest, immunohistochemical detection of CD31 and GFP revealed that many of these cells originated from the endothelium of the microvascular fragments (Figs. 2fh). However, vessel segments with a lumen surrounded by endothelial cells were not detectable anymore.

To analyse in more detail the progressive vessel dedifferentiation throughout the pre-cultivation period of 28 days, we stained microvascular fragments inside scaffolds, which were pre-cultivated for $0,3,7,14$ and 28 days, with antibodies against the endothelial cell marker CD31 and the perivascular cell marker $\alpha$-SMA (Figs. 3a-e). By this, we could demonstrate that the fragments continuously lost their $\alpha$-SMA-positive perivascular cell coverage over time. In parallel, we detected a progressive disorganisation of the endothelial cells. Additionally, we analysed the cell viability within the scaffolds at day 3, 7, 14 and 28 (Figs. 3f-i). At each time point, we detected a comparably low number of trypan blue-positive dead cells within the samples (3 d: $8 \pm 3 \%$; 7 d: $5 \pm 2 \%$; 14 d: $7 \pm 3 \%$; 28 d: $4 \pm 2 \%$ ), indicating that the cultivation conditions did not markedly affect their viability.

\section{Vascularisation of implanted scaffolds in vivo}

Based on our in vitro observation of a continuous vessel regression throughout the pre-cultivation period of 28 days we decided to implant only freshly seeded scaffolds as controls and scaffolds, which were pre-cultivated for 28 days, into the dorsal skinfold chamber to characterise the effects of these two extreme conditions on the in vivo vascularisation of the implants.

Directly after implantation into the dorsal skinfold chamber of C57BL/6 wild-type mice, individual GFPpositive microvascular fragments could be easily detected 

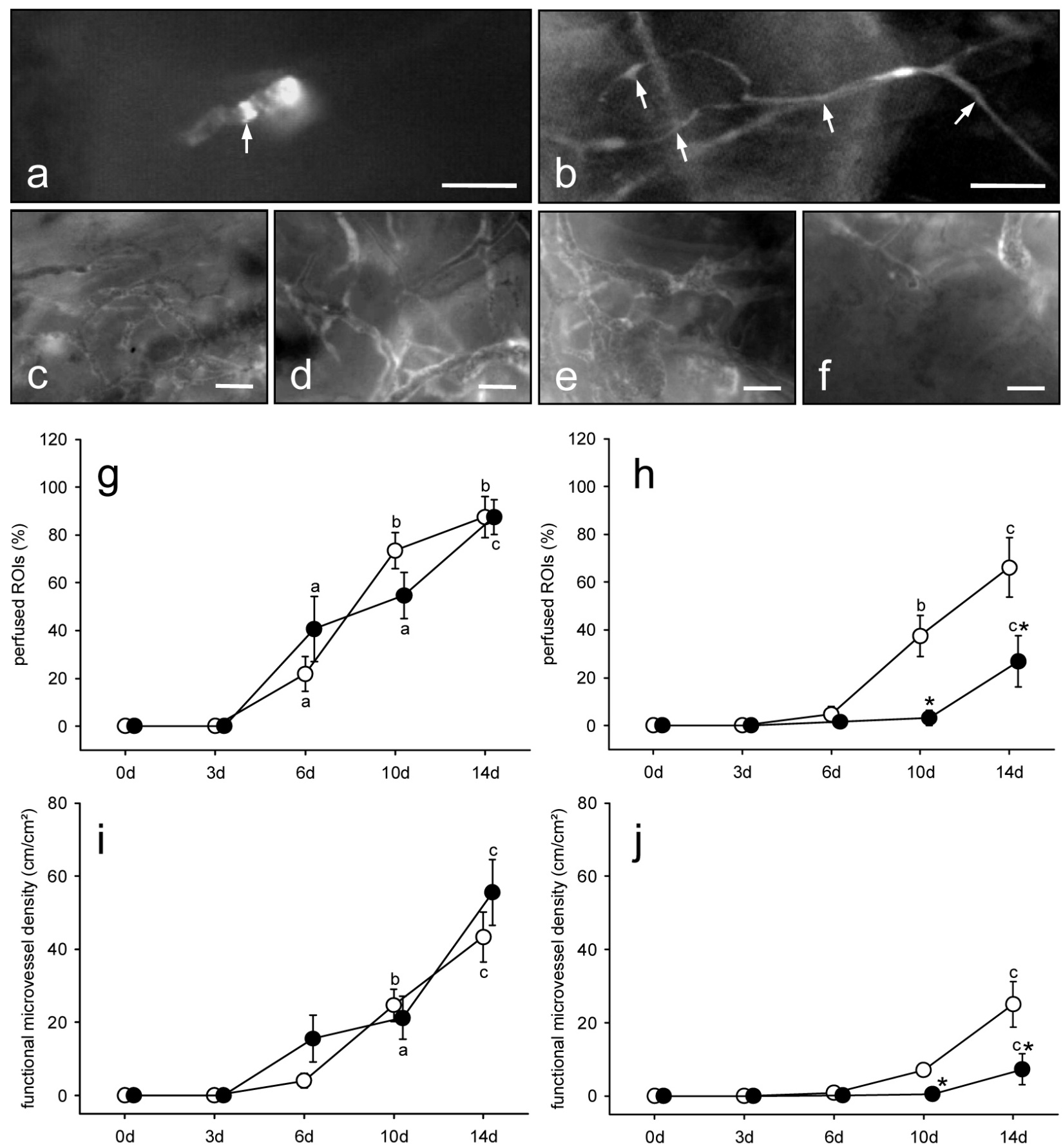

Fig. 4. a, b: Intravital fluorescence microscopy of a freshly seeded control scaffold (a) and a pre-cultivated scaffold (b) directly after implantation into the dorsal skinfold chamber of a C57BL/6 mouse. In the control scaffold, a GFPpositive microvascular fragment (a, arrow) is visualised on the surface in blue-light epi-illumination. In contrast, the surface of the pre-cultivated scaffold is covered by a network of interconnected GFP-positive cells with filigrane cell protuberances (b, arrows). Scale bars: $40 \mu \mathrm{m}$. c-f: Intravital fluorescence microscopy in blue-light epi-illumination with contrast enhancement by $5 \%$ FITC-labelled dextran 150,000 i.v. of the border (c, d) and centre (e, f) of a freshly seeded control scaffold (c, e) and a pre-cultivated scaffold (d, f) at day 14 after implantation into the dorsal skinfold chamber. Note that the control scaffold exhibits a markedly improved vascularisation in the centre (e) when compared to the pre-cultivated implant (f). Scale bars: $55 \mu \mathrm{m}$. g-j: Perfused ROIs (\%) (g, h) and functional microvessel density $(\mathbf{i}, \mathbf{j})$ within the border $(\mathbf{g}, \mathbf{i})$ and the centre $(\mathbf{h}, \mathbf{j})$ of freshly seeded control scaffolds (white circles, $n=8$ ) and pre-cultivated scaffolds (black circles, $n=8$ ) after implantation into dorsal skinfold chambers, as assessed by intravital fluorescence microscopy and computer-assisted image analysis. Means \pm SEM. ${ }^{a} p<0.05 v s .0 \mathrm{~d}$ and $3 \mathrm{~d}$ within each individual group; ${ }^{b} p<0.05 v s .0 \mathrm{~d}, 3 \mathrm{~d}$ and $6 \mathrm{~d}$ within each individual group; ${ }^{c} p<0.05 v s .0 \mathrm{~d}, 3 \mathrm{~d}, 6 \mathrm{~d}$ and $10 \mathrm{~d}$ within each individual group; $* p<0.05 \mathrm{vs}$. control. 

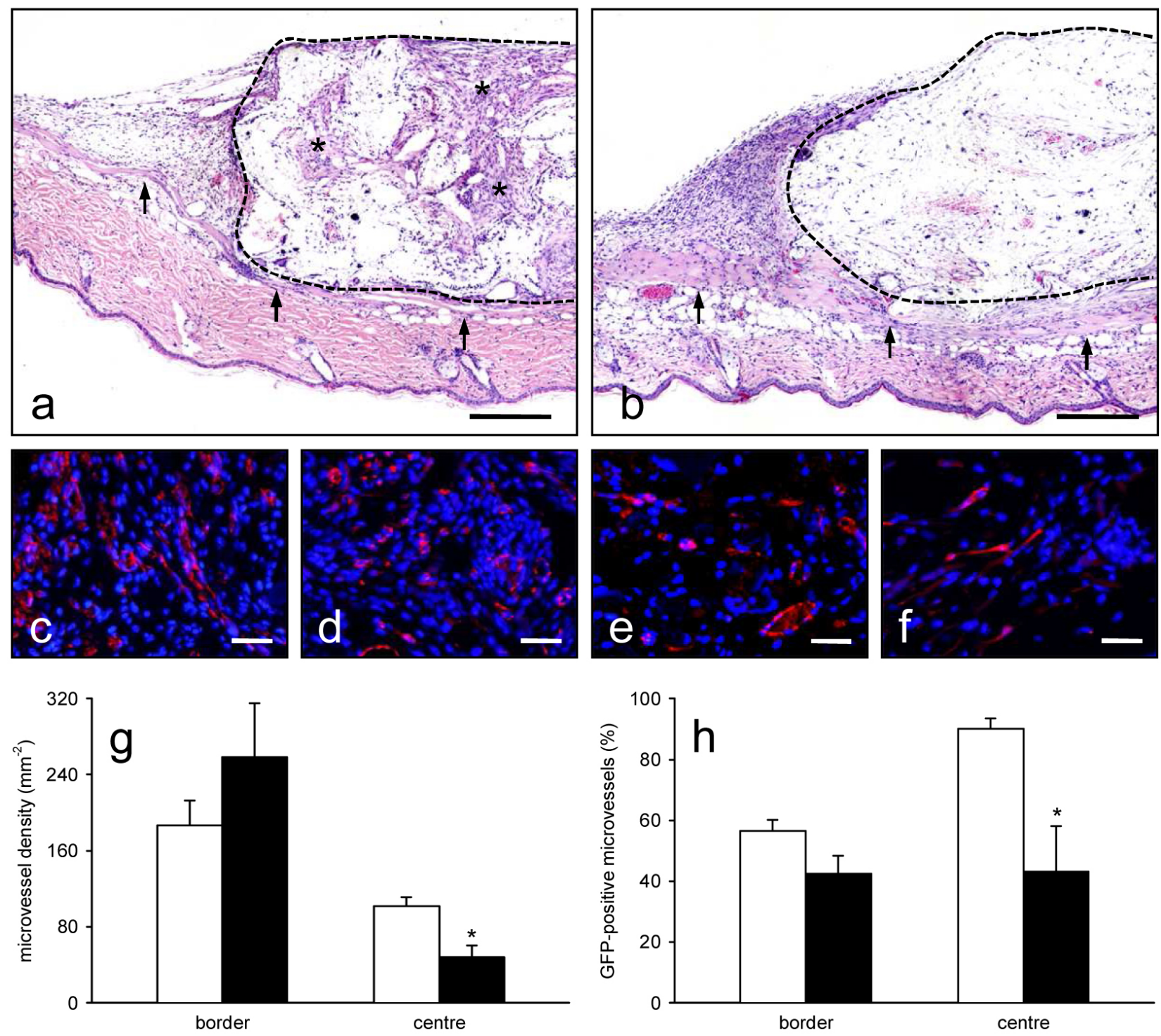

Fig. 5. a, b: HE-stained sections of a freshly seeded control scaffold (a, borders marked by broken line) and a precultivated scaffold (b, borders marked by broken line) at day 14 after implantation onto the host striated muscle tissue (arrows) within the dorsal skinfold chamber of C57BL/6 mice. Note that most of the pores of freshly seeded control scaffolds are filled with a dense, newly formed granulation tissue (a, asterisks), whereas the pores in precultivated implants solely contain loosely distributed cells. Scale bars: $320 \mu \mathrm{m}$. c-f: Immunohistochemical detection of CD31-positive microvessels (red) in the border $(\mathbf{c}, \mathbf{d})$ and centre $(\mathbf{e}, \mathbf{f})$ of a freshly seeded control scaffold $(\mathbf{c}, \mathbf{e})$ and pre-cultivated scaffold $(\mathbf{d}, \mathbf{f})$ at day 14 . The sections are stained with Hoechst 33342 to identify cell nuclei. Scale bars: $40 \mu \mathrm{m}$. g, h: Microvessel density $\left(\mathrm{mm}^{-2}\right)(\mathbf{g})$ and GFP-positive microvessels (h) within the border and centre of freshly seeded control (white bars, $n=8$ ) and pre-cultivated scaffolds (black bars, $n=8$ ) at day 14 after implantation into dorsal skinfold chambers, as assessed by immunohistochemical analysis. Means \pm SEM $* p<0.05 v s$. control.

on the surface of freshly seeded control scaffolds by means of intravital fluorescence microscopy (Fig. 4a). In line with our histological analyses, the surface of pre-cultivated scaffolds was covered by a network of interconnected GFP-positive cells with filigrane cell protuberances (Fig. 4b). Despite this different morphology, both scaffold types induced a comparable angiogenic host tissue response in their border zones (Figs. 4c, d, g and i). This was characterised by the formation of vessel sprouts, which progressively grew from the surrounding host microvasculature into the implants and finally interconnected with each other to form new microvascular networks.
In contrast, the vascularisation of the implants' centre markedly differed between freshly seeded and precultivated scaffolds. As already described in our previous study (Laschke et al., 2012), freshly isolated microvascular fragments are able to proliferate and interconnect with each other and with ingrowing host vessels over time. This process could not be observed in pre-cultivated implants. Accordingly, freshly seeded control scaffolds exhibited an improved vascularisation in their centre, as indicated by a significantly higher number of perfused ROIs and increased functional microvessel density between days 10 and 14 when compared to pre-cultivated scaffolds (Figs. $4 e, f, h$ and $j)$. 


\section{Incorporation and angioarchitecture of implanted scaffolds}

At the end of the in vivo experiments, i.e. day 14 after implantation into the dorsal skinfold chamber, the scaffolds were additional analysed by histology and immunohistochemistry. By this, we could demonstrate that most of the pores of freshly seeded control scaffolds were filled with a dense, newly formed granulation tissue, whereas the pores of implants, which were pre-cultivated for 28 days, solely contained a few, loosely distributed cells (Figs. 5a and b). This indicates a better incorporation of freshly seeded control scaffolds.

In addition, we found that the granulation tissue in the centre of control scaffolds presented with a higher density of CD31-positive microvessels when compared to precultivated implants (Figs. 5c-g). Of interest, we detected GFP-positive microvessels in the border and centre zones of both scaffold types. However, the fraction of these vessels was significantly reduced to $\sim 40 \%$ in the centre of pre-cultivated implants when compared to controls ( $90 \%)$ (Fig. 5h).

\section{Discussion}

Prevascularisation of tissue constructs is a promising strategy for the rapid establishment of an adequate blood supply after their implantation into a defect site. For the generation of preformed microvascular networks inside scaffolds, various in vitro and in situ approaches have been suggested so far (Rivron et al., 2008; Laschke and Menger, 2012; Baiguera and Ribatti, 2013). However, although these approaches have produced good results in experimental studies, they are difficult to translate into clinical practice, because they are associated with complex and time-consuming cell isolation and cultivation procedures or require repetitive surgical interventions. Accordingly, there is currently a clear trend in the field of tissue engineering towards simplified solutions, which primarily use the self-regenerative capacity of the human body.

Adipose tissue-derived microvascular fragments represent promising vascularisation units. In contrast to single cell isolates from the stromal fraction of fat tissue (Scherberich et al., 2010), these fragments bear the major advantage that they already consist of fully functional arteriolar, capillary and venular vessel segments. Under clinical conditions, these fragments may be isolated from a patient by minimally-invasive liposuction and directly transferred to a defect site in an intra-operative one-step procedure. Recently, we and others reported that these fragments possess the capacity to reassemble into fully functional microvascular networks (Shepherd et al., 2004; Shepherd et al., 2007; Laschke et al., 2012). This, however, requires several days during which the cells inside implanted tissue constructs are still exposed to hypoxia and, thus, may not survive. In the present study, we demonstrate now that this critical phase cannot be shortened by pre-cultivation of fragment-seeded scaffolds prior to their implantation.
For our experiments, we used the identical type of polyurethane scaffolds, as in former studies, focusing on the establishment of novel vascularisation strategies for tissue engineering (Laschke et al., 2010b; Laschke et al., 2011b; Laschke et al., 2013). These scaffolds have originally been designed for bone tissue engineering and, thus, contain nanosize hydroxyapatite particles to improve their osteoconductivity (Laschke et al., 2010a). Due to their elastic properties and their high porosity they are particularly suitable for the dynamic seeding with individual cells (Duttenhoefer et al., 2013), cell spheroids (Laschke et al., 2013; Laschke et al., 2014b) and microvascular fragments (Laschke et al., 2012; Laschke et al., 2014a).

We found that the pre-cultivation of microvascular fragments inside porous polyurethane scaffolds for 28 days results in the loss of their native vessel morphology without markedly affecting their viability. In fact, precultivated fragments continuously lost their stabilising $\alpha$-SMA-positive perivascular cell coverage over time. In parallel, we detected a progressive disorganisation of their endothelial cells, finally resulting in the loss of vessel lumina and the formation of a network of individual endothelial cells with filigrane cell protuberances, which grew along the scaffold strands. This observed vessel dedifferentiation is in line with previous studies, in which microvascular fragments or in situ generated microvascular networks partly lost their $\alpha$-SMA-positive perivascular cell layers during short-term cultivation of 3-10 days (Shepherd et al., 2004; Laschke et al., 2011b). The interaction of endothelial cells and these stabilising cell layers, in turn, is important for maintaining long-term microvascular integrity (Díaz-Flores et al., 1991; Lindahl et al., 1997). In addition, the lack of blood perfusion during cultivation may have further contributed to the regression of individual microvascular fragments, because blood flow-induced mechanical forces have been shown to regulate endothelial cell proliferation and survival (Kadohama et al., 2007).

When we initially planned our study, we chose a precultivation period of 28 days, because such a time frame may offer the possibility to differentiate mesenchymal stem cells, which are an important component of microvascular fragments (Laschke et al., 2012; McDaniel et al., 2014), into tissue-specific cells prior to their transfer into a defect site. Our results now show that in vitro differentiation of mesenchymal stem cells does not contribute to the development of a functional microvascular network. With the benefit of hindsight, it is furthermore clear that a markedly shortened pre-cultivation period results in less vessel regression. However, our in vitro analyses showed that even such a short pre-cultivation period of 3-7 days is already associated with a significant loss of vessel morphology and perivascular cell stabilisation and, in addition, does not lead to our primary goal, i.e. the formation of fully functional microvascular networks inside the scaffolds.

In contrast to our results, others (Hoying et al., 1996; Shepherd et al., 2004; Shepherd et al., 2007) have shown that microvascular fragments can spontaneously reassemble in vitro to form microvascular networks. This 
may be explained by the fact that they embedded the fragments in a collagen gel, which provided a specific environment similar to the extracellular matrix (ECM) of physiological tissues. During recent years, it has become evident that this ECM not only serves as a mechanical framework for newly developing microvessels, but also stimulates intracellular signalling pathways, which are crucially involved in the process of angiogenesis (Ingber, 2002; Yang et al., 2004). Accordingly, we speculate that optimising the in vitro culture conditions by additional filling of the scaffold pores with an ECM equivalent may have improved the outcome in the present experimental setting. This approach may also bear the advantage that the ECM equivalent can be supplemented with angiogenic growth factors to promote angiogenesis and network formation inside the construct (Laschke et al., 2008). On the other hand, it has to be considered that each additional modification of a tissue construct makes it more difficult to introduce it into clinical practice due to significant technical, regulatory and policy hurdles (Stegemann et $a l ., 2014)$. Seen from that point of view, we feel that the implantation of scaffolds seeded with freshly isolated, non-cultivated microvascular fragments is currently the most realistic strategy for potential clinical applications.

In line with our histomorphological characterisation of pre-cultivated and freshly seeded control scaffolds, intravital fluorescence microscopy revealed that both scaffold types exhibited a different vascularisation pattern after implantation into the dorsal skinfold chamber. While a comparable number of microvessels developed in the border zones of the implants, freshly seeded scaffolds presented with a markedly improved vascularisation in their centre when compared to pre-cultivated ones. Our finding that $90 \%$ of all microvessels inside freshly seeded scaffolds stained positive for GFP indicates that this improved vascularisation was mainly caused by the reassembly of microvascular fragments into functional microvascular networks over time. This also resulted in higher tissue content inside the implants, further emphasising the importance of a sufficient vascularisation for the survival and successful incorporation of tissue constructs (Laschke et al., 2006; Auger et al., 2013).

Despite a deteriorated vascularisation we still detected GFP-positive microvessels in the border and centre zones of pre-cultivated scaffolds at day 14 after implantation. This is an interesting result, which indicates that the endothelial cells of the original microvascular fragments still contributed to the development of a new microvasculature inside the implants, even after the long pre-cultivation period of 28 days. The comparable vascularisation in the border zones of pre-cultivated and control scaffolds may be best interpreted as an unspecific angiogenic host tissue response due to the vitalisation with cells. In fact, both implant types were vitalised with a mixture of different cells, which suffered from hypoxia during the initial phase after implantation and, thus, may have stimulated the host tissue by the release of angiogenic growth factors, such as VEGF. In line with this view, Schumann et al. (2009) demonstrated that the vascularisation of scaffolds seeded with osteoblast-like cells, bone marrow mesenchymal stem cells or a combination of both cell types is VEGF-related rather than dependent on the specific vessel-forming capacity of individual cells inside the implants.

\section{Conclusions}

The present animal study indicates that pre-cultivation of adipose tissue-derived microvascular fragments from mice inside scaffolds does not improve their in vivo vascularisation capacity, but markedly affects their physiological vessel morphology and function. If the same holds true for human adipose tissue-derived microvascular fragments, this means that freshly isolated fragments should be preferred as vascularisation units. This notion is supported by the fact that intra-operative one-step procedures with minimal in vitro manipulations are preferred for clinical tissue engineering applications.

\section{Acknowledgements}

This work was supported by the Large Bone Defect Healing Program of AO Foundation. We are grateful for the excellent technical assistance of Janine Becker and Julia Parakenings.

\section{References}

Auger FA, Gibot L, Lacroix D (2013) The pivotal role of vascularization in tissue engineering. Annu Rev Biomed Eng 15: 177-200.

Baiguera S, Ribatti D (2013) Endothelialization approaches for viable engineered tissues. Angiogenesis 16: 1-14.

Díaz-Flores L, Gutiérrez R, Varela H, Rancel N, Valladares F (1991) Microvascular pericytes: a review of their morphological and functional characteristics. Histol Histopathol 6: 269-286.

Duttenhoefer F, Lara de Freitas R, Meury T, Loibl M, Benneker LM, Richards RG, Alini M, Verrier S (2013) 3D scaffolds co-seeded with human endothelial progenitor and mesenchymal stem cells: evidence of prevascularisation within 7 days. Eur Cell Mater 26: 49-64.

Güven S, Mehrkens A, Saxer F, Schaefer DJ, Martinetti R, Martin I, Scherberich A (2011) Engineering of large osteogenic grafts with rapid engraftment capacity using mesenchymal and endothelial progenitors from human adipose tissue. Biomaterials 32: 5801-5809.

Hiscox AM, Stone AL, Limesand S, Hoying JB, Williams SK (2008) An islet-stabilizing implant constructed using a preformed vasculature. Tissue Eng Part A 14: 433440 .

Hoying JB, Boswell CA, Williams SK (1996) Angiogenic potential of microvessel fragments established in three-dimensional collagen gels. In Vitro Cell Dev Biol Anim 32: 409-419.

Ingber DE (2002) Mechanical signaling and the cellular response to extracellular matrix in angiogenesis and cardiovascular physiology. Circ Res 91: 877-887. 
Kadohama T, Nishimura K, Hoshino Y, Sasajima T, Sumpio BE (2007) Effects of different types of fluid shear stress on endothelial cell proliferation and survival. J Cell Physiol 212: 244-251.

Klar AS, Güven S, Biedermann T, Luginbühl J, Böttcher-Haberzeth S, Meuli-Simmen C, Meuli M, Martin I, Scherberich A, Reichmann E (2014) Tissue-engineered dermo-epidermal skin grafts prevascularized with adiposederived cells. Biomaterials 35: 5065-5078.

Laschke MW, Menger MD (2012) Vascularization in tissue engineering: angiogenesis versus inosculation. Eur Surg Res 48: 85-92.

Laschke MW, Harder Y, Amon M, Martin I, Farhadi J, Ring A, Torio-Padron N, Schramm R, Rücker M, Junker D, Häufel JM, Carvalho C, Heberer M, Germann G, Vollmar $\mathrm{B}$, Menger MD (2006) Angiogenesis in tissue engineering: breathing life into constructed tissue substitutes. Tissue Eng 12: 2093-2104.

Laschke MW, Rücker M, Jensen G, Carvalho C, Mülhaupt R, Gellrich NC, Menger MD (2008) Incorporation of growth factor containing Matrigel promotes vascularization of porous PLGA scaffolds. J Biomed Mater Res A 85: 397-407.

Laschke MW, Vollmar B, Menger MD (2009) Inosculation: connecting the life-sustaining pipelines. Tissue Eng Part B Rev 15: 455-465.

Laschke MW, Strohe A, Menger MD, Alini M, Eglin D (2010a) In vitro and in vivo evaluation of a novel nanosize hydroxyapatite particles/poly(ester-urethane) composite scaffold for bone tissue engineering. Acta Biomater 6: 2020-2027.

Laschke MW, Mussawy H, Schuler S, Eglin D, Alini M, Menger MD (2010b) Promoting external inosculation of prevascularised tissue constructs by pre-cultivation in an angiogenic extracellular matrix. Eur Cell Mater 20: 356-366.

Laschke MW, Vollmar B, Menger MD (2011a) The dorsal skinfold chamber: window into the dynamic interaction of biomaterials with their surrounding host tissue. Eur Cell Mater 22: 147-164.

Laschke MW, Mussawy H, Schuler S, Kazakov A, Rücker M, Eglin D, Alini M, Menger MD (2011b) Shortterm cultivation of in situ prevascularized tissue constructs accelerates inosculation of their preformed microvascular networks after implantation into the host tissue. Tissue Eng Part A 17: 841-853.

Laschke MW, Kleer S, Scheuer C, Schuler S, Garcia P, Eglin D, Alini M, Menger MD (2012) Vascularisation of porous scaffolds is improved by incorporation of adipose tissue-derived microvascular fragments. Eur Cell Mater 24: 266-277.

Laschke MW, Schank TE, Scheuer C, Kleer S, Schuler S, Metzger W, Eglin D, Alini M, Menger MD (2013) Threedimensional spheroids of adipose-derived mesenchymal stem cells are potent initiators of blood vessel formation in porous polyurethane scaffolds. Acta Biomater 9: 68766884.

Laschke MW, Grässer C, Kleer S, Scheuer C, Eglin D, Alini M, Menger MD (2014a) Adipose tissue-derived microvascular fragments from aged donors exhibit an impaired vascularisation capacity. Eur Cell Mater 28: 287-298.

Laschke MW, Schank TE, Scheuer C, Kleer S, Shadmanov T, Eglin D, Alini M, Menger MD (2014b) In vitro osteogenic differentiation of adipose-derived mesenchymal stem cell spheroids impairs their in vivo vascularization capacity inside implanted porous polyurethane scaffolds. Acta Biomater 10: 4226-4235.

Lindahl P, Johansson BR, Levéen P, Betsholtz C (1997) Pericyte loss and microaneurysm formation in PDGF-Bdeficient mice. Science 277: 242-245.

Matsuda K, Falkenberg KJ, Woods AA, Choi YS, Morrison WA, Dilley RJ (2013) Adipose-derived stem cells promote angiogenesis and tissue formation for in vivo tissue engineering. Tissue Eng Part A 19: 1327-1335.

McDaniel JS, Pilia M, Ward CL, Pollot BE, Rathbone CR (2014) Characterization and multilineage potential of cells derived from isolated microvascular fragments. J Surg Res 192: 214-222.

Müller AM, Mehrkens A, Schäfer DJ, Jaquiery C, Güven S, Lehmicke M, Martinetti R, Farhadi I, Jakob M, Scherberich A, Martin I (2010) Towards an intraoperative engineering of osteogenic and vasculogenic grafts from the stromal vascular fraction of human adipose tissue. Eur Cell Mater 19: 127-135.

Novosel EC, Kleinhans C, Kluger PJ (2011) Vascularization is the key challenge in tissue engineering. Adv Drug Deliv Rev 63: 300-311.

Nunes SS, Krishnan L, Gerard CS, Dale JR, Maddie MA, Benton RL, Hoying JB (2010a) Angiogenic potential of microvessel fragments is independent of the tissue of origin and can be influenced by the cellular composition of the implants. Microcirculation 17: 557-567.

Nunes SS, Greer KA, Stiening CM, Chen HY, Kidd KR, Schwartz MA, Sullivan CJ, Rekapally H, Hoying JB (2010b) Implanted microvessels progress through distinct neovascularization phenotypes. Microvasc Res 79: 10-20.

Okabe M, Ikawa M, Kominami K, Nakanishi T, Nishimune Y (1997) 'Green mice' as a source of ubiquitous green cells. FEBS Lett 407: 313-319.

Phelps EA, García AJ (2010) Engineering more than a cell: vascularization strategies in tissue engineering. Curr Opin Biotechnol 21: 704-709.

Pilia M, McDaniel JS, Guda T, Chen XK, Rhoads RP, Allen RE, Corona BT, Rathbone CR (2014) Transplantation and perfusion of microvascular fragments in a rodent model of volumetric muscle loss injury. Eur Cell Mater 28: 11-23.

Rivron NC, Liu J J, Rouwkema J, de Boer J, van Blitterswijk CA (2008) Engineering vascularised tissues in vitro. Eur Cell Mater 15: 27-40.

Rohringer S, Hofbauer P, Schneider KH, Husa AM, Feichtinger G, Peterbauer-Scherb A, Redl H, Holnthoner W (2014) Mechanisms of vasculogenesis in 3D fibrin matrices mediated by the interaction of adipose-derived stem cells and endothelial cells. Angiogenesis 17: 921-933.

Scherberich A, Galli R, Jaquiery C, Farhadi J, Martin I (2007) Three-dimensional perfusion culture of human adipose tissue-derived endothelial and osteoblastic progenitors generates osteogenic constructs with intrinsic vascularization capacity. Stem Cells 25: 1823-1829. 
Scherberich A, Müller AM, Schäfer DJ, Banfi A, Martin I (2010) Adipose tissue-derived progenitors for engineering osteogenic and vasculogenic grafts. J Cell Physiol 225: 348-353.

Schumann P, Tavassol F, Lindhorst D, Stuehmer C, Bormann KH, Kampmann A, Mülhaupt R, Laschke MW, Menger MD, Gellrich NC, Rücker M (2009) Consequences of seeded cell type on vascularization of tissue engineering constructs in vivo. Microvasc Res 78: 180-190.

Shepherd BR, Chen HY, Smith CM, Gruionu G, Williams SK, Hoying JB (2004) Rapid perfusion and network remodeling in a microvascular construct after implantation. Arterioscler Thromb Vasc Biol 24: 898-904.

Shepherd BR, Hoying JB, Williams SK (2007) Microvascular transplantation after acute myocardial infarction. Tissue Eng 13: 2871-2879.

Stegemann JP, Verrier S, Gebhard F, Laschke MW, Martin I, Simpson H, Miclau T (2014) Cell therapy for bone repair: narrowing the gap between vision and practice. Eur Cell Mater 27: 1-4.

Yang B, Cao DJ, Sainz I, Colman RW, Guo YL (2004) Different roles of ERK and p38 MAP kinases during tube formation from endothelial cells cultured in 3-dimensional collagen matrices. J Cell Physiol 200: 360-369.

\section{Discussion with Reviewers}

Reviewer I: The experimental plan and conditions tested are not very clear. Did authors compare all different preand post- implantation time points?

Authors: We repetitively characterised in vitro the changes in morphology and stability of microvascular fragments during a pre-cultivation period of 28 days. For this purpose, fragment-seeded scaffolds were analysed by histology and immunohistochemistry directly after the seeding procedure (control) as well as at day 3, 7, 14 and 28. We found that there is a progressive change in vessel morphology, which is associated with the loss of a stabilising perivascular cell layer and finally ends at day 28 in a network of thin, elongated endothelial cells, which grow along the scaffold strands. Based on this observation of a continuous vessel regression over time we decided to implant only freshly seeded scaffolds as controls and scaffolds, which were precultivated for 28 days, into the dorsal skinfold chamber to characterise the effects of these two extreme conditions on the in vivo vascularisation of the implants.

Reviewer I: The data in this manuscript are not sufficient to claim that the pre-culture of microvascular fragments does not improve in vivo vascularisation. Characterisation of in vitro stability of microvascular fragments should be performed.

Authors: We performed additional immunohistochemical analyses to characterise the in vitro stability of microvascular fragments throughout the pre-cultivation period of 28 days. For this purpose, microvascular fragments in freshly seeded control scaffolds as well as in scaffolds that were pre-cultivated for 3, 7, 14 and 28 days, were stained with antibodies against the endothelial cell marker CD31 and the perivascular cell marker $\alpha$-smooth muscle actin (SMA). By this, we could demonstrate that the fragments continuously lost their $\alpha$-SMA-positive perivascular cell coverage over time. In parallel, we detected a progressive disorganisation of the endothelial cells, finally resulting in the loss of vessel lumina and the formation of a network of individual endothelial cells with filigrane cell protuberances, which grew along the scaffold strands.

Reviewer I: What is the rationale for pre-cultivating the isolated microvascular fragments for such long time (28 days)? Improving in vitro culture conditions may preserve the function and capacity of microvascular fragments. Also earlier pre-cultivation time points may result in better in vivo vascularisation.

Authors: When we initially planned our study, we chose a pre-cultivation period of 28 days, because such a time frame may offer the possibility to differentiate mesenchymal stem cells, which are an important component of microvascular fragments (Laschke et al., 2012; McDaniel et al., 2014), into tissue-specific cells prior to their transfer into a defect site. Our results now show that in vitro differentiation of mesenchymal stem cells does not contribute to the development of a functional microvascular network. With the benefit of hindsight, it is furthermore clear that a markedly shortened pre-cultivation period results in less vessel regression. However, our in vitro analyses showed that even such a short pre-cultivation period of 3-7 days is already associated with a significant loss of vessel morphology and perivascular cell stabilisation and, in addition, does not lead to our primary goal, i.e. the formation of fully functional microvascular networks inside the scaffolds.

Reviewer II: Discuss the choice of nano-sized hydroxyapatite particles / poly (ester-urethane) as the biomaterial in this approach.

Authors: We used the identical type of polyurethane scaffolds as in former studies focusing on the establishment of novel vascularisation strategies for tissue engineering (Laschke et al., 2010b; Laschke et al., 2011b; Laschke et al., 2013). These scaffolds were originally designed for bone tissue engineering and, thus, contain nanosize hydroxyapatite particles to improve their osteoconductivity (Laschke et al., 2010a). Due to their elastic properties and their high porosity they are particularly suitable for the dynamic seeding with individual cells (Duttenhoefer et al., 2013), cell spheroids (Laschke et al., 2013; Laschke et al., 2014b) and microvascular fragments (Laschke et al., 2012; Laschke et al., 2014a).

Reviewer II: Regarding the in vivo experiments, the authors used fragments derived from GFP transgenic mice with intravital fluorescence microscopy using fluorescein isothiocyanate (FITC)-labelled dextran, arguing that it is possible to see enhanced contrast inside the perfused vessels. Why did the authors not use a different animal model, such as an RFP transgenic mice [B6.Cg-Tg (CAGDsRed * MST) 1Nagy / J] from the Jackson Laboratory or other mice that allow better evaluation of the perfusion levels by contrast between the endothelial cells, that are red, and the FITC labelled dextran? 
Authors: We agree with the reviewer that it is elegant to detect endothelial cells and blood perfusion simultaneously by means of different fluorescence spectra during intravital fluorescence microscopy. Certainly, we will test this approach in one of our future studies. However, the present study is directly based on a previous study (Laschke et al., 2012), in which we demonstrated for the first time improved vascularisation of polyurethane scaffolds by seeding them with GFP-positive microvascular fragments. Accordingly, we chose the identical experimental setting, which had already been well established and, thus, also allows for a better comparability of the results. 